\title{
Spin-Dependent Fabry-Pérot Interference from a Cu Thin Film Grown on fcc Co(001)
}

\author{
Y.Z. Wu, ${ }^{1}$ A. K. Schmid, ${ }^{2}$ M. S. Altman, ${ }^{3}$ X. F. Jin, ${ }^{4,5}$ and Z. Q. Qiu ${ }^{1}$ \\ ${ }^{1}$ Department of Physics, University of California, Berkeley, California 94720, USA \\ ${ }^{2}$ NCEM, Lawrence Berkeley National Laboratory, Berkeley, California 94720, USA \\ ${ }^{3}$ Department of Physics, Hong Kong University of Science and Technology, Hong Kong SAR, People's Republic of China \\ ${ }^{4}$ Department of Physics, Fudan University, Shanghai, People's Republic of China \\ ${ }^{5}$ International Center of Quantum Structures, Chinese Academy of Science, People's Republic of China
}

(Received 17 May 2004; published 18 January 2005)

\begin{abstract}
Spin-dependent electron reflection from a $\mathrm{Cu}$ thin film grown on $\mathrm{Co} / \mathrm{Cu}(001)$ was investigated using spin-polarized low-energy electron microscopy (SPLEEM). Fabry-Pérot type interference was observed and is explained using the phase accumulation model. SPLEEM images of the $\mathrm{Cu}$ overlayer reveal magnetic domains in the Co underlayer, with the domain contrast oscillating with electron energy and $\mathrm{Cu}$ film thickness. This behavior is attributed to the spin-dependent electron reflectivity at the $\mathrm{Cu} / \mathrm{Co}$ interface which leads to spin-dependent Fabry-Pérot electron interference in the $\mathrm{Cu}$ film.
\end{abstract}

DOI: 10.1103/PhysRevLett.94.027201

PACS numbers: 75.70.Ak, 68.37.Nq, 75.75.+a

Quantum well states (QWS) that are formed by electron confinement in thin metallic films can dramatically influence film properties and result in many interesting phenomena, such as film stability [1] and oscillatory magnetic interlayer coupling [2-4], etc. Photoemission methods provide the most direct information on QWS below the vacuum level. A key result is that electron quantization caused by confinement can be well described by the phase accumulation model (PAM) [5]. On the contrary, phenomena related to unoccupied QWS above the vacuum level have been explored to a considerably lesser extent. This is especially true for spin-dependent phenomena in magnetic nanostructures, which is due, in part, to the low efficiency of spin-resolved photoemission measurements. Although energy levels above the vacuum level are not occupied by electrons and thus do not contribute directly to the electronic energies, injection of hot electrons into these unoccupied states can provide unique information on material properties. For example, hot electron injection has been applied for a long time in techniques such as lowenergy electron diffraction and low-energy electron microscopy [6]. The spin degree of freedom of hot electrons was also successfully exploited to study spin-dependent properties of materials by several different methods, such as imaging of magnetic domain structure using scanning electron microscopy with polarization analysis [7] and spin-polarized low-energy electron microscopy (SPLEEM) [8]. Since both occupied and unoccupied electronic states are quantized, modification of the unoccupied states above the vacuum level by confinement can have a significant effect on electron scattering measurements that are based on hot electron injection. For example, oscillations of the reflected electron intensity from metallic thin films are known to occur with varying incident electron energy due to a quantum size effect [9-11]. A similar interference effect was also observed in electron transmission into metallic multilayers [12]. Recently, a spin selective electron interferometer was demonstrated by electron reflectivity measurements from thin metallic films $[13,14]$. SPLEEM measurements of quantum well resonances have also been used to derive spin-resolved unoccupied energy bands of $\mathrm{Fe}$ films [15]. These discoveries suggest that a spin-dependent quantum size effect involving unoccupied states above vacuum $[16,17]$ could bring new capabilities to electron microscopy techniques if the spin degree of freedom of hot electrons is integrated into spectroscopic measurements. In this Letter, we report on development of this new approach in a SPLEEM study of $\mathrm{Cu}$ thin films grown on $\mathrm{fcc} \mathrm{Co} / \mathrm{Cu}(001)$. We find that the electron reflectivity from the $\mathrm{Cu}$ thin film exhibits FabryPérot type interference: when the $\mathrm{Cu}$ thickness increases, the energies of the interference peaks decrease (or increase) for $k$ vector greater (or less) than half of the Brillouin zone (BZ) vector. This interference effect results in a spin asymmetry of the electron reflectivity from the $\mathrm{Cu}$ film. SPLEEM images of the $\mathrm{Cu}$ film reveal the magnetic domain structure of the underlying Co film, with domain contrast oscillating with electron energy and $\mathrm{Cu}$ film thickness. This observation is attributed to the spin-dependent reflectivity at the $\mathrm{Cu} / \mathrm{Co}$ interface.

The experiment was performed in the SPLEEM instrument at Lawrence Berkeley National Laboratory, which operates at a base pressure of $5 \times 10^{-11}$ Torr. $\mathrm{A} \mathrm{Cu}(001)$ single crystal substrate was cleaned by cycles of $\mathrm{Ar}$ ion sputtering at $1.5-3 \mathrm{keV}$ energy and annealing at $\sim 1000 \mathrm{~K}$. After cleaning, no surface contamination was detectable using Auger electron spectroscopy. All films were deposited at room temperature, and the pressure remained below $2 \times 10^{-10}$ Torr during deposition. In the SPLEEM, a spinpolarized low-energy electron beam is directed at the sample surface at normal incidence, and the specular beam is magnified in an electron-optical column to form either a real-space image of the sample or a backscattered electron diffraction pattern. The SPLEEM can be used for 
convenient and very precise film-thickness control during in situ film growth by monitoring average image intensity oscillations [18] in a similar manner as reflected high energy electron diffraction oscillations. A 5 ML (monolayer) $\mathrm{Co}$ film was grown first on the $\mathrm{Cu}(001)$ surface to serve as a $\mathrm{Co}$ substrate, and then $\mathrm{Cu}$ films were grown while simultaneously recording SPLEEM images and repeatedly scanning the incident electron energy to provide a systematic thickness and energy dependent study. Because of the instrument limitation, each energy scan covers only a range of $\sim 13 \mathrm{eV}$.

The reflectivity of elastically scattered unpolarized electrons from the $\mathrm{Cu} / \mathrm{Co} / \mathrm{Cu}(001)$ specimen was determined from the laterally averaged and spin-integrated SPLEEM image intensity. After normalizing the data by the intensity spectrum obtained from a thick $\mathrm{Cu}$ film that does not exhibit quantum interference, resonance reflectivity peaks are clearly observed whose energy decreases or increases with increasing $\mathrm{Cu}$ film thickness for electron energy below or above $\sim 19 \mathrm{eV}$, respectively [Figs. 1(a) and 1(b)]. To understand the resonance peaks of the electron reflectivity, we consider electron reflection from a $\mathrm{Cu}$ thin film on an fcc Co substrate. If the complex reflectivities of the electron wave in $\mathrm{Cu}$ at the $\mathrm{Cu} /$ vacuum and $\mathrm{Cu} / \mathrm{Co}$ interfaces are $r_{B} \exp \left(i \phi_{B}\right)$ and $r_{C} \exp \left(i \phi_{C}\right)$, respectively, where $r$ and $\phi$ denote the magnitude and phase gain of the electron reflection at the corresponding interface, it is straightforward to derive the following expression for the total electron reflectivity by satisfying the continuity boundary conditions at the two $\mathrm{Cu}$ interfaces:

$$
R=\frac{r_{B}^{2}+r_{C}^{2}+2 r_{B} r_{C} \cos \left(2 k d_{\mathrm{Cu}}+\phi_{B}+\phi_{C}\right)}{1+r_{B}^{2} r_{C}^{2}+2 r_{B} r_{C} \cos \left(2 k d_{\mathrm{Cu}}+\phi_{B}+\phi_{C}\right)} .
$$

Here $d_{\mathrm{Cu}}$ is the $\mathrm{Cu}$ film thickness and $k$ is the electron

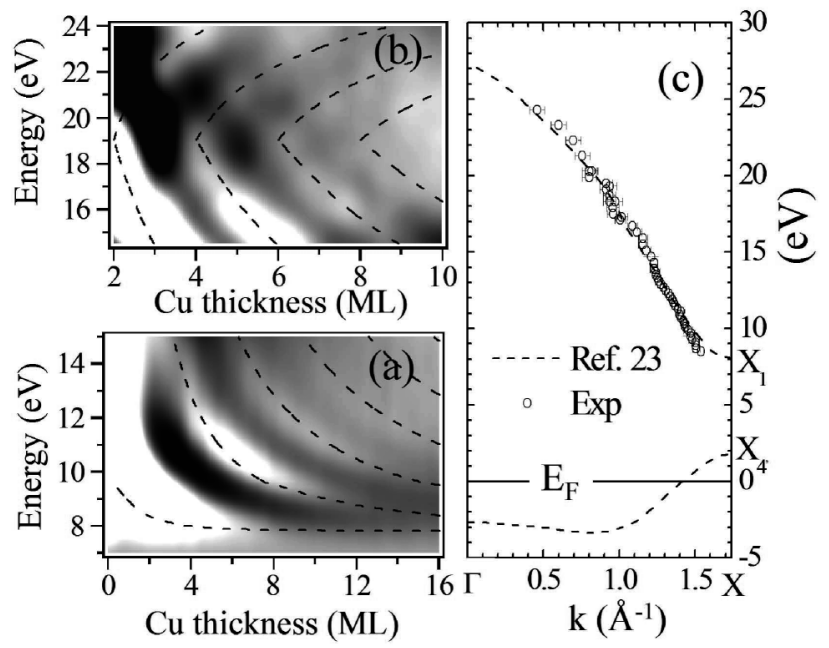

FIG. 1. Electron reflectivity from $\mathrm{Cu} / \mathrm{Co} / \mathrm{Cu}(001)$. The dashed lines in (a) and (b) are theoretical calculations based on the phase accumulation model. (c) Experimental and theoretical results for the $\mathrm{Cu}$ energy band. momentum vector in the $\mathrm{Cu}$ film. Equation (1) simply describes a classical Fabry-Pérot interferometer. It is interesting to note that the maximum electron reflectivity takes place at the interference condition of

$$
2 k d_{\mathrm{Cu}}+\phi_{C}+\phi_{B}=2 \pi n, \quad n=\text { integer, }
$$

which is exactly the result of the phase accumulation model [5] for QWS below the vacuum level. Therefore the phase accumulation model describes both the confined QWS below the vacuum level and the interference condition above the vacuum level. In a solid, an electron also experiences the lattice periodic potential which generates an envelope function for the electron Bloch wave [19]. Thus Eq. (2) should be valid for $k<k_{\mathrm{BZ}} / 2$ where $k_{\mathrm{BZ}}$ is the $\mathrm{BZ}$ wave vector. For $k>k_{\mathrm{BZ}} / 2$, Eq. (2) should be rewritten as

$$
2\left(k_{\mathrm{BZ}}-k\right) d_{\mathrm{Cu}}-\phi_{C}-\phi_{B}=2 \pi \nu, \quad n=\text { integer. }
$$

Although Eqs. (2) and (3) are mathematically equivalent at integer $\mathrm{Cu}$ thickness, they have different physical meanings. While Eq. (2) describes the QWS for $k<k_{\mathrm{BZ}} / 2$ with the index $n$ being the number of nodes of the electron wave function, Eq. (3) describes the electron envelope function at $k>k_{\mathrm{BZ}} / 2$ with the index $\nu$ being the number of nodes of the envelope function. The envelope function arises from a beating effect between the electron wave and the periodic lattice potential at $k>k_{\mathrm{BZ}} / 2$ [5]. Therefore Eq. (3) actually represents the quantization condition for the envelope function [19-21]. One important consequence, as proposed by Egger et al. [13], is that Eqs. (2) and (3) should lead to an opposite energy vs thickness dispersion for QWS at $k<k_{\mathrm{BZ}} / 2$ and $k>k_{\mathrm{BZ}} / 2$ with a crossover occurring at $k=k_{\mathrm{BZ}} / 2$. In the energy range of $8-26 \mathrm{eV}$ above $E_{F}$, there is one relevant energy band along the [001] direction $(\Gamma X)$ with $\Delta_{1}$ symmetry, in which the energy decreases with increasing $k$ and crosses the $k=k_{\mathrm{BZ}} / 2$ point at $\sim 19 \mathrm{eV}[22,23]$. Therefore, for energy greater or less than $19 \mathrm{eV}$, the Fabry-Pérot interference condition should be determined by Eq. (2) or Eq. (3), respectively, leading to a corresponding increase or decrease of the QWS energy with increasing $\mathrm{Cu}$ thickness, respectively. Such a crossover effect is now unambiguously confirmed by our experimental observation shown in Fig. 1(b). In particular, the $2 \mathrm{ML}$ oscillation periodicity of the $\mathrm{Cu}$ film thickness occurs as expected at the crossover energy of $19 \mathrm{eV}$, which corresponds exactly to the $k=k_{\mathrm{BZ}} / 2$ point.

To quantitatively explain the result of Fig. 1, we calculated the QWS using the phase accumulation model. The reflection phases for hot electrons at the upper and lower interface of the $\mathrm{Cu}$ film are calculated as $\phi_{B}=0$ and $\phi_{C}=\pi-2 \arcsin \sqrt{\left(E-E_{L}\right) /\left(E_{U}-E_{L}\right)}$, where $E_{U}=$ $9.6 \mathrm{eV}$ and $E_{L}=2.5 \mathrm{eV}$ are the upper and lower edges of the Co energy gap $[24,25]$. Adopting the Cu energy band from band structure calculations [23], we can use the PAM 
to calculate the resonance conditions of the QWS as a function of energy and $\mathrm{Cu}$ film thickness. The result of the PAM calculation, plotted as dashed lines in Figs. 1(a) and 1(b), shows excellent agreement with the experimental data. At a given energy, the periodicity of the reflectivity oscillations as a function of $\mathrm{Cu}$ film thickness determines the corresponding momentum vector; thus the $\mathrm{Cu}$ energy band can be derived from the experimental data of Figs. 1(a) and 1(b) [15]. Figure 1(c) shows a comparison of the $\mathrm{Cu}$ energy band determined from this experiment (open circles) with the band structure calculation result (dashed line).

After understanding the interference effect of unpolarized electrons in the $\mathrm{Cu}$ film, we turn our attention to the spin-dependent reflectivity. Adjusting the incident electron spin polarization from a direction that is parallel $(p)$ to one that is antiparallel ( $a p)$ to the sample magnetization causes the electron reflectivity to change. Figure 2(a) shows representative spin-resolved electron reflectivity measurements of $\mathrm{Cu} / \mathrm{Co}(5 \mathrm{ML}) / \mathrm{Cu}(001)$ as a function of $\mathrm{Cu}$ film thickness at two incident electron energies. The reflectivity peaks are due to the quantum interference identified in Fig. 1. The electron reflectivity of the $\mathrm{Cu}$ film is clearly spin dependent. This effect was attributed previously to the phase difference of the electron reflection at the $\mathrm{Cu} / \mathrm{Co}$ interface [13], which can account for the peak position. However, we find that both the positions and the magnitudes of the reflectivity peaks depend on the incident electron spin direction. This indicates that the ferromag-

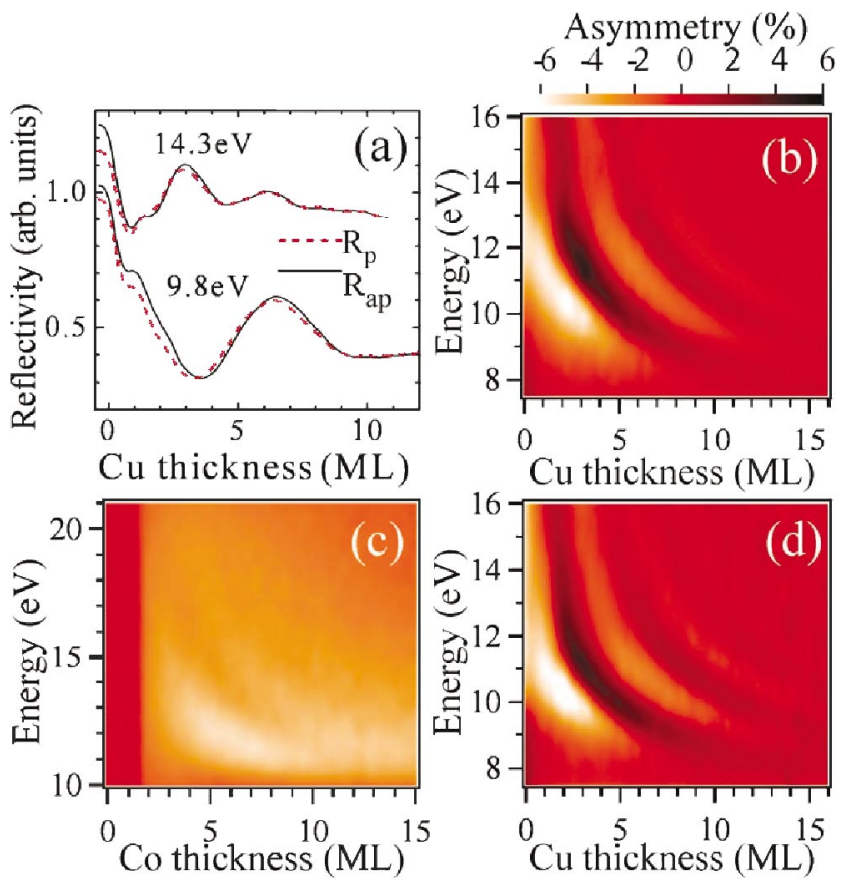

FIG. 2 (color). (a) Spin-dependent electron reflectivity from $\mathrm{Cu} / \mathrm{Co}(5 \mathrm{ML}) / \mathrm{Cu}(001)$. Asymmetry of the spin-dependent electron reflectivity from (b) $\mathrm{Cu} / \mathrm{Co}(5 \mathrm{ML}) / \mathrm{Cu}(001)$, (c) $\mathrm{Co} / \mathrm{Cu}(001)$, and (d) $\mathrm{Cu} / \mathrm{Co}(15 \mathrm{ML}) / \mathrm{Cu}(001)$. netic Co generates not only a spin-dependent phase shift, but also causes a spin dependence of the magnitude of the electron reflectivity at the $\mathrm{Cu} / \mathrm{Co}$ interface. In other words, both $r_{C}$ and $\phi_{C}$ in Eq. (1) are spin dependent. By measuring the difference of the $p$-spin and $a p$-spin reflectivity, we obtained the asymmetry of the electron reflectivity, $\left(R_{p}-R_{a p}\right) /\left(R_{p}+R_{a p}\right)$ [Fig. 2(b)]. It is seen that the spin-dependent reflectivity at the $\mathrm{Cu} / \mathrm{Co}$ interface results in a spin-dependent Fabry-Pérot interference from the $\mathrm{Cu}$ film with the asymmetry oscillating as a function of both the electron energy and the $\mathrm{Cu}$ film thickness. Since the Co film is in the ultrathin regime, we also studied the electron reflection asymmetry in $\mathrm{Co} / \mathrm{Cu}(001)$ as a function of Co film thickness and electron energy [Fig. 2(c)]. For Co thinner than $1.6 \mathrm{ML}$, the asymmetry is zero because the $\mathrm{Co} / \mathrm{Cu}(001)$ film is paramagnetic at room temperature. For Co thicker than 1.6 ML, the reflection asymmetry exhibits a weak interference effect. Comparing Figs. 2(b) and 2(c), we notice several differences. First, the sign of the asymmetry for $\mathrm{Cu} / \mathrm{Co}(5 \mathrm{ML}) / \mathrm{Cu}(001)$ alternates between positive and negative, while the sign of the asymmetry for $\mathrm{Co} / \mathrm{Cu}(001)$ remains unchanged. Second, the asymmetry oscillation amplitude of $\mathrm{Cu} / \mathrm{Co}(5 \mathrm{ML}) /$ $\mathrm{Cu}(001)$ is an order of magnitude greater than that of $\mathrm{Co} / \mathrm{Cu}(001)$. Thus we conclude that the interference shown in Fig. 2(b) is dominated by the $\mathrm{Cu}$ film. This conclusion is further supported by the asymmetry measurement for $\mathrm{Cu} / \mathrm{Co}(15 \mathrm{ML}) / \mathrm{Cu}(001)$ [Fig. 2(d)], which shows a result that is almost identical to the result for $\mathrm{Cu} / \mathrm{Co}(5 \mathrm{ML}) / \mathrm{Cu}(001)$ [Fig. 2(b)].

To further explore the spin-dependent Fabry-Pérot interference, we performed SPLEEM measurements of both $\mathrm{Co}(5 \mathrm{ML}) / \mathrm{Cu}(001)$ and $\mathrm{Cu} / \mathrm{Co}(5 \mathrm{ML}) / \mathrm{Cu}(001)$ at an identical sample position. For $\mathrm{Co}(5 \mathrm{ML}) / \mathrm{Cu}(001)$ [Fig. 3(a)], the domain intensity (dark and bright) remains unchanged with increasing electron energy, which agrees with the result of Fig. 2(c) that the sign of the asymmetry does not change for $\mathrm{Co} / \mathrm{Cu}(001)$. For $\mathrm{Cu} / \mathrm{Co}(5 \mathrm{ML}) /$ $\mathrm{Cu}(001)$, we obtained SPLEEM images at different electron energies with fixed $\mathrm{Cu}$ thickness [Fig. 2(b)] and at different $\mathrm{Cu}$ thicknesses with fixed electron energy [Fig. 2(c)]. In both cases, we observed not only the magnetic domains of the Co layer buried beneath the $\mathrm{Cu}$ film but also domain contrast reversals [dark-bright switching as compared with the fixed domain contrast of $\mathrm{Co} / \mathrm{Cu}(001)]$. This result further confirms the spindependent nature of Fabry-Pérot interference in the $\mathrm{Cu}$ film and highlights that both the magnitude and the sign of the electron reflection asymmetry oscillate with the electron energy and the $\mathrm{Cu}$ film thickness.

In summary, we have investigated the spin-dependent electron reflectivity from a $\mathrm{Cu}$ thin film grown on $\mathrm{Co} / \mathrm{Cu}(001)$ using SPLEEM. The Fabry-Pérot interference peak energy was found to increase or decrease with the $\mathrm{Cu}$ thickness for an electron wave vector less than or greater 
(a)
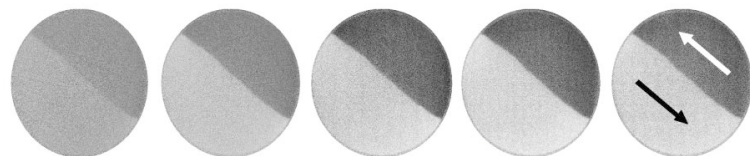

(b)
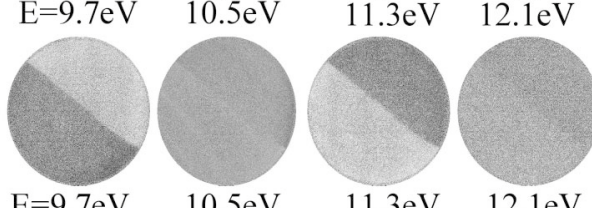

$13.9 \mathrm{eV}$

(c)
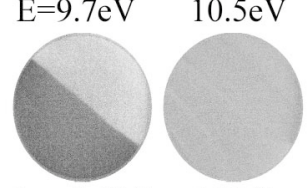

$\mathrm{d}_{\mathrm{Cu}}=3.6 \mathrm{ML}$

4.8ML
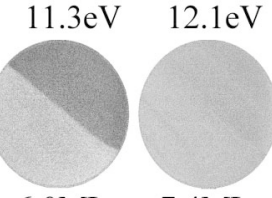

$6.0 \mathrm{ML}$
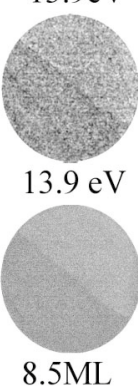

FIG. 3. SPLEEM images of (a) $\mathrm{Co}(5 \mathrm{ML}) / \mathrm{Cu}(001)$ at different electron energies, (b) $\mathrm{Cu}(6.0 \mathrm{ML}) / \mathrm{Co}(5 \mathrm{ML}) / \mathrm{Cu}(001)$ at different energies, and (c) $\mathrm{Cu} / \mathrm{Co}(5 \mathrm{ML}) / \mathrm{Cu}(001)$ at different $\mathrm{Cu}$ thicknesses and fixed $11.3 \mathrm{eV}$ electron energy. The oscillation of the domain contrast (both magnitude and sign) in (b) and (c) shows the spin-dependent Fabry-Pérot interference of the $\mathrm{Cu}$ film. The arrows in (a) indicate the Co magnetization directions. The image field of the view is $7 \mu \mathrm{m}$. All images correspond to the same sample area.

than half of the BZ vector, respectively. The phase accumulation model successfully explains the experimental observations. Moreover, the spin-dependent electron reflectivity at the $\mathrm{Cu} / \mathrm{Co}$ interface results in a spin-dependent Fabry-Pérot interference in the $\mathrm{Cu}$ film, making it possible to detect magnetic domains in the Co underlayer in SPLEEM images of the $\mathrm{Cu}$ overlayer film.

This work was supported by National Science Foundation DMR-0405259, U.S. Department of Energy DE-AC03-76SF00098, Chinese National Science Foundation, Chinese Science and Technology Ministry for 973 Project, and ICQS of CAS.

[1] D.-A. Luh, T. Miller, J. J. Paggel, M. Y. Chou, and T.-C. Chiang, Science 292, 1131 (2001).

[2] S. S. P. Parkin, N. More, and K. P. Roche, Phys. Rev. Lett. 64, 2304 (1990).
[3] J.E. Ortega and F. J. Himpsel, Phys. Rev. Lett. 69, 844 (1992).

[4] R. K. Kawakami, E. Rotenberg, Ernesto J. EscorciaAparicio, Hyuk J. Choi, J.H. Wolfe, N. V. Smith, and Z. Q. Qiu, Phys. Rev. Lett. 82, 4098 (1999).

[5] N. V. Smith, N. B. Brookes, Y. Chang, and P. D. Johnson, Phys. Rev. B 49, 332 (1994).

[6] E. Bauer, Rep. Prog. Phys. 57, 895 (1994).

[7] M. R. Scheinfein, J. Unguris, M.H. Kelley, D. T. Pierce, and R. J. Celotta, Rev. Sci. Instrum. 61, 2501 (1990).

[8] T. Duden and E. Bauer, J. Electron Microsc. 47, 379 (1998).

[9] R. E. Thomas, J. Appl. Phys. 41, 5330 (1970).

[10] R. C. Jaklevic and John Lambe, Phys. Rev. B 12, 4146 (1975).

[11] B. T. Jonker, N. C. Bartelt, and R. L. Park, Surf. Sci. 127, 183 (1983).

[12] Qi-Gao Zhu, Yunong Yang, Ellen D. Williams, and Robert L. Park, Phys. Rev. Lett. 59, 835 (1987).

[13] S. Egger, C. H. Back, J. Krewer, and D. Pescia, Phys. Rev. Lett. 83, 2833 (1999).

[14] T. Scheunemann, R. Feder, J. Henk, E. Bauer, T. Duden, H. Pinkvos, H. Poppa, and K. Wurm, Solid State Commun. 104, 787 (1997).

[15] R. Zdyb and E. Bauer, Phys. Rev. Lett. 88, 166403 (2002).

[16] K. Garrison, Y. Chang, and P. D. Johnson, Phys. Rev. Lett. 71, 2801 (1993).

[17] D. H. Yu, M. Donath, J. Braun, and G. Rangelov, Phys. Rev. B 68, 155415 (2003).

[18] K. L. Man, H. Poppa, and M. S. Altman, Surf. Sci. 480, 163 (2001).

[19] Z. Q. Qiu and N. V. Smith, J. Phys. Condens. Matter 14, R169 (2002).

[20] T. C. Chiang, Surf. Sci. Rep. 39, 183 (2000).

[21] R. K. Kawakami, E. Rotenberg, Hyuk J. Choi, Ernesto J. Escorcia-Aparicio, M. O. Bowen, J.H. Wolfe, E. Aronholtz, Z. Zhang, N. V. Smith, and Z.Q. Qiu, Nature (London) 398, 132 (1999).

[22] R. Courths and S. Hüfner, Phys. Rep. 112, 53 (1984).

[23] R. Lässer, N. V. Smith, and R. L. Benbow, Phys. Rev. B 24, 1895 (1981).

[24] G. J. Mankey, R. F. Willis, and F. J. Himpsel, Phys. Rev. B 48, 10284 (1993).

[25] P. Van Gelderen, S. Crampin, and J. E. Inglesfield, Phys. Rev. B 53, 9115 (1996). 